$02,05,11,12$

\title{
Конденсация (псевдо)магнонов в двумерной анизотропной $S=1$ (псевдо)спиновой системе
}

\author{
(C) Е.В. Васинович, А.С. Москвин, Ю.Д. Панов \\ Уральский фредеральный университет, \\ Екатеринбург, Россия \\ E-mail: e.vasinovich@gmail.com
}

\begin{abstract}
В рамках (псевдо)спинового формализма была изучена двумерная анизотропная система $S=1$-центров типа зарядовых триплетов в системах с переменной валентностью или систем „полужестких“ бозонов с ограничением на заполнение узлов решетки $n=0,1,2$. В предположении основного состояния типа квантового парамагнетика с помощью метода швингеровских бозонов найден спектр псевдоспиновых волн, а также условия конденсации псевдомагнонов с фазовым переходом в сверхпроводящее состояние.
\end{abstract}

Работа выполнена при поддержке Программы 211 Правительства Российской Федерации, соглашение № 02.А03.21.0006, и проектов № 2277 и № 5719 Министерства образования и науки Российской Федерации.

DOI: $10.21883 /$ FTT.2018.11.46647.05NN

\section{1. Введение}

В отличие от квантовых магнетиков со спином $1 / 2$ системы со спином $S=1$ характеризуются более сложным гамильтонианом с появлением одноионной анизотропии, биквадратичных межцентровых взаимодействий и принципиально новых фазовых состояний типа квантового парамагнетика. Интерес к таким системам, в частности, связан с описанием сильноанизотропных магнетиков на основе $\mathrm{Ni}^{2+}(S=1)$. Еще в 70-е годы был изучен двумерный гейзенберговский антиферромагнетик $\mathrm{K}_{2} \mathrm{NiF}_{4}$ [1]. В 80-е годы был проведен ряд работ по квазиодномерным системам, включая $\mathrm{CsNiCl}_{3}$ [2], который имеет слабую анизотропию вдоль одной оси, $\mathrm{CsFeBr}_{3}$ [3], который обладает сильной плоскостной анизотропией. Различные системы на основе ионов $\mathrm{Ni}^{2+}$, например, $\left[\mathrm{Ni}\left(\mathrm{HF}_{2}\right)(3-\mathrm{Clpy})_{4}\right] \mathrm{BF}_{4}[4,5]$ и $\mathrm{NiCl}_{2} 4 \mathrm{SC}\left(\mathrm{NH}_{2}\right)_{2}$ [6], активно изучаются по сей день.

Помимо сильноанизотропных магнетиков, интерес к $S=1$-системам связан и с так называемыми псевдоспиновыми системами типа „полужестких“ (semi-hard-core) бозонов с ограничением на заполнение узлов решетки $n=0,1,2$, или системами ионов со смешанной валентностью типа „триплета“ $\mathrm{Cu}^{1+, 2+, 3+}$ в купратах $\mathrm{La}_{2-x} \mathrm{Sr}_{x} \mathrm{CuO}_{4}, \mathrm{Bi}^{3+, 4+, 5+}$ в висмутатах $[7,8,9,10]$.

Использование теоретических методов, хорошо зарекомендовавших себя при изучении квантовых магнетиков со спином 1/2, таких как методы точной диагонализации $[11,12]$, разложение в ряд [13], ренормгруппы [14], функции Грина [15], для систем со спином $S=1$ сталкивается, прежде всего, с существенно более сложным гамильтонианом с появлением одноионной анизотропии, биквадратичных межцентровых взаимодействий и новыми фазовыми состояниями. Достаточно эффективным в описании $S=1$-систем представляется метод швингеровских бозонов [16], который был впервые реализован для двумерного антиферромагнетика со спином $S=1$ в работе [17], но для простейшего гамильтониана. В данной работе этот метод развит нами для более сложных псевдоспиновых систем с учетом эффектов биквадратичной двухцентровой анизотропии.

\section{2. Модель зарядовых триплетов: псевдоспиновый формализм}

Мы ограничимся рассмотрением модельных 2D-псевдоспиновых систем типа „полужестких“ бозонов с ограничением на заполнение узлов решетки $n=0,1,2$, или систем ионов со смешанной валентностью типа „триплета“ $\mathrm{Cu}^{1+, 2+, 3+}$ в купратах или $\mathrm{Bi}^{3+, 4+, 5+}$ в висмутатах $[7,8,9,10]$, связывая зарядовый триплет с тремя состояниями псевдоспина $S=1$ и используя известные методы описания спиновых систем. Так, упрощенная модель зарядовых триплетов в квазидвумерных купратах предполагает полное пренебрежение спиновыми и орбитальными степенями свободы. Три зарядовых состояния кластеров $\mathrm{CuO}_{4}$ в $\mathrm{CuO}_{2}$-Плоскостях, номинально соответствующих $\mathrm{Cu}^{2+}, \mathrm{Cu}^{3+}, \mathrm{Cu}^{1+}$, мы сопоставляем с тремя проекциями псевдоспина $S=1$ с $M_{S}=0,+1,-1$ соответственно.

Спиновая алгебра $S=1\left(M_{S}=0, \pm 1\right)$ включает восемь независимых нетривиальных операторов (три дипольных и пять квадрупольных)

$$
S_{z} ; S_{ \pm}=\mp \frac{1}{\sqrt{2}}\left(S_{x} \pm i S_{y}\right) ; S_{z}^{2} ; T_{ \pm}=\left\{S_{z}, S_{ \pm}\right\} ; S_{ \pm}^{2} .
$$

Операторы повышения/понижения $S_{ \pm}$и $T_{ \pm}$меняют проекцию псевдоспина на \pm 1 , но различным образом: $\left\langle 0\left|S_{ \pm}\right| \mp 1\right\rangle=\left\langle \pm 1\left|S_{ \pm}\right| 0\right\rangle=\mp 1, \quad\left\langle 0\left|T_{ \pm}\right| \mp 1\right\rangle=$ $=-\left\langle \pm 1\left|T_{ \pm}\right| 0\right\rangle=+1$. Вместо этих операторов удобно использовать комбинированные операторы $P_{ \pm}=$ $=\frac{1}{2}\left(S_{ \pm}+T_{ \pm}\right)$и $N_{ \pm}=\frac{1}{2}\left(S_{ \pm}-T_{ \pm}\right)$, которые соответственно описывают переходы $|0\rangle \leftrightarrow|+1\rangle$ и $|0\rangle \leftrightarrow|-1\rangle$. Операторы повышения/понижения $S_{ \pm}^{2}$ описывают переходы $|-1\rangle \leftrightarrow|+1\rangle$. Локальный (узельный) недиагональный параметр порядка $\left\langle S_{ \pm}^{2}\right\rangle$ отличен от нуля только 
если на узле имеется суперпозиция состояний $|+1\rangle$ и $|-1\rangle$.

Псевдоспиновый формализм позволяет в наиболее общем виде описать эффекты переноса, а также эффекты локальных и нелокальных корреляций в системе зарядовых триплетов [7]. Запишем эффективный гамильтониан, который коммутирует с Z-компонентой полного псевдоспина $\sum_{i} S_{i z}$ и, таким образом, сохраняет полный заряд системы, как сумму потенциальной и кинетической энергий

$$
\begin{gathered}
H=H_{\mathrm{pot}}+H_{\mathrm{kin}}^{(1)}+H_{\mathrm{kin}}^{(2)}, \\
H_{\mathrm{pot}}=\sum_{i}\left(\Delta S_{i z}^{2}-\mu S_{i z}\right)+\frac{1}{2} V \sum_{\langle i j\rangle} S_{i z} S_{j z}, \\
H_{\mathrm{kin}}^{(1)}=\frac{1}{2} \sum_{\langle i j\rangle}\left[t^{p} P_{i+} P_{j-}+t^{n} N_{i+} N_{j-}\right. \\
\left.+\frac{1}{2} t^{p n}\left(P_{i+} N_{j-}+N_{i+} P_{j-}\right)+\text { h.c. }\right], \\
H_{\mathrm{kin}}^{(2)}=-\frac{1}{2} t^{b} \sum_{\langle i j\rangle}\left(S_{i+}^{2} S_{j-}^{2}+S_{i-}^{2} S_{j+}^{2}\right) .
\end{gathered}
$$

За исключением некоторых слагаемых, неинвариантных относительно обращения времени в (4), этот гамильтониан представляет один из наиболее общих анизотропных $S=1$ спин-гамильтонианов. Первое слагаемое в (3), или „одноионная анизотропия“, описывает корреляционные эффекты плотность-плотность на узлах. Второе слагаемое может быть связано с псевдомагнитным полем вдоль $Z$-оси, либо с химическим потенциалом относительно добавления новых частиц. Последний член описывает межузельные взаимодействия (корреляции) типа плотность-плотность. Гамильтониан (4) (ХҮ-анизотропия) описывает одночастичный перенос в системе; транспорт РР-типа отвечает за переходы вида $|0\rangle+|+1\rangle \leftrightarrow|+1\rangle+|0\rangle$, NN-типа отвечает за $|0\rangle+|-1\rangle \leftrightarrow|-1\rangle+|0\rangle$, а РN-типа реализует $|0\rangle+|0\rangle \leftrightarrow| \pm\rangle+|\mp\rangle$. Гамильтониан (5) (биквадратичная двухцентровая анизотропия) описывает двухчастичный перенос.

В зависимости от соотношения между параметрами гамильтониана (2) и величины полного заряда основное состояние системы соответствует либо однородной непроводящей фазе типа квантового парамагнетика с $\left\langle S_{z}\right\rangle=\left\langle S_{z}^{2}\right\rangle=0$, реализуемой при больших положительных значениях корреляционного параметра $\Delta$, либо непроводящей фазе зарядового упорядочения $(\mathrm{CO})$ - аналогу антиферромагнитного упорядочения вдоль $Z$-оси, либо вариантам сверхпроводящих XY-(SF, superfluid) фаз с отличными от нуля параметрами порядка типа $\left\langle S_{ \pm}\right\rangle$и/или $\left\langle S_{ \pm}^{2}\right\rangle$, сопровождаемых однородным ферро-упорядочением, или неоднородным антиферро-упорядочением (supersolid phase) $Z$-компонент псевдоспина.

Наряду с температурными фазовыми переходами, гамильтониан (2) представляет большие возможности исследования квантовых фазовых переходов, связанных, в частности, с изменением корреляционного параметра $\Delta$ (одноионной анизотропии) $[17,18,19,20,21]$.

Псевдоспин-гамильтониан (2) имеет унверсальный вид для систем любой размерности. Ниже мы остановимся на анализе элементарных возбуждений псевдоспиновой $S=1$-системы - псевдомагнонов на квадратной решетке в предположении основного состояния типа квантового парамагнетика. Практический интерес к такой задаче связан с проблемой индуцирования высокотемпературной сверхпроводимости в родительских купратах, основное состояние которых соответствует валентности меди $\mathrm{Cu}^{2+}$, что в рамках псевдоспинового формализма как раз соответствует состоянию квантового парамагнетика с $\left\langle S_{z}\right\rangle=\left\langle S_{z}^{2}\right\rangle=0$.

\section{3. Представление швингеровских бозонов}

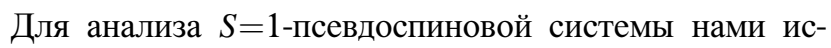
пользовалось представление швингеровских бозонов в среднем поле [16,17]. В этом методе трем проекциям псевдоспина $S=1$ сопоставляются три бозе-оператора рождения/уничтожения квазичастиц над вакуумом

$$
|1\rangle=b_{+}^{\dagger}|v\rangle,|0\rangle=b_{0}^{\dagger}|v\rangle,|-1\rangle=b_{-}^{\dagger}|v\rangle,
$$

при выполнении условия

$$
b_{+}^{\dagger} b_{+}+b_{0}^{\dagger} b_{0}+b_{-}^{\dagger} b_{-}=1 .
$$

Заменяя спиновые операторы в (2) на бозонные

$$
\begin{gathered}
P_{+}=-b_{+}^{\dagger} b_{0}, \quad P_{-}=-P_{+}^{\dagger}=b_{0}^{\dagger} b_{+}, \\
N_{+}=-b_{0}^{\dagger} b_{-}, \quad N_{-}=-N_{+}^{\dagger}=b_{-}^{\dagger} b_{0}, \\
S_{z}=b_{+}^{\dagger} b_{+}-b_{-}^{\dagger} b_{-}, \quad S_{z}^{2}=b_{+}^{\dagger} b_{+}+b_{-}^{\dagger} b_{-}, \\
S_{+}^{2}=b_{+}^{\dagger} b_{-}, \quad S_{-}^{2}=\left(S_{+}^{2}\right)^{\dagger}=b_{-}^{\dagger} b_{+},
\end{gathered}
$$

мы получим гамильтониан следующего вида

$$
\begin{aligned}
H_{\mathrm{pot}}= & \Delta \sum_{i}\left(b_{i+}^{\dagger} b_{i+}+b_{i-}^{\dagger} b_{i-}\right)-\mu \sum_{i}\left(b_{i+}^{\dagger} b_{i+}-b_{i-}^{\dagger} b_{i-}\right) \\
& +\frac{V}{2} \sum_{\langle i j\rangle}\left(b_{i+}^{\dagger} b_{i+}-b_{i-}^{\dagger} b_{i-}\right)\left(b_{j+}^{\dagger} b_{j+}-b_{j-}^{\dagger} b_{j-}\right) \\
& -v \sum_{i}\left(b_{i+}^{\dagger} b_{i+}+b_{i-}^{\dagger} b_{i-}+b_{0}^{2}-1\right) \\
& H_{\mathrm{kin}}^{(1)}=-\frac{b_{0}^{2}}{2} \sum_{\langle i j\rangle}\left[t^{p} b_{i+}^{\dagger} b_{j+}+t^{n} b_{i-}^{\dagger} b_{j-}\right. \\
& \left.+\frac{1}{2} t^{p n}\left(b_{i+}^{\dagger} b_{j-}^{\dagger}+b_{i-}^{\dagger} b_{j+}^{\dagger}\right)+\text { h.c. }\right] \\
H_{\mathrm{kin}}^{(2)}= & -\frac{t^{b}}{2} \sum_{\langle i j\rangle}\left(b_{i+}^{\dagger} b_{i-} b_{j-}^{\dagger} b_{j+}+b_{i-}^{\dagger} b_{i+} b_{j+}^{\dagger} b_{j-}\right)
\end{aligned}
$$

где учтено основное (вакуумное) состояние квантового парамагнетика, соответствующее конденсату бозонов $b_{0}$, 
то есть $\left\langle b_{0}\right\rangle=\left\langle b_{0}^{\dagger}\right\rangle=b_{0}$. Введенный параметр $v$ обеспечивает выполнение ограничения (7).

Квадратичные слагаемые в гамильтониане линеаризуются в среднем поле следующим образом:

$$
\begin{aligned}
& b_{i+}^{\dagger} b_{i-} b_{j-}^{\dagger} b_{j+}+b_{i-}^{\dagger} b_{i+} b_{j+}^{\dagger} b_{j-}=q\left(b_{i+}^{\dagger} b_{i-}+b_{j-}^{\dagger} b_{j+}+\text { h.c. }\right) \\
& -2 q^{2},\left(b_{i+}^{\dagger} b_{i+}-b_{i-}^{\dagger} b_{i-}\right)\left(b_{j+}^{\dagger} b_{j+}-b_{j-}^{\dagger} b_{j-}\right) \\
& =\frac{1}{2}\left(1-b_{0}^{2}+m\right)\left(b_{i+}^{\dagger} b_{i+}+b_{j+}^{\dagger} b_{j+}\right) \\
& +\frac{1}{2}\left(1-b_{0}^{2}-m\right)\left(b_{i-}^{\dagger} b_{i-}+b_{j-}^{\dagger} b_{j-}\right) \\
& -p\left(b_{i+} b_{j-}+b_{i-} b_{j+}+\text { h.c. }\right)+2 p^{2}-\frac{1}{2}\left(1-b_{0}^{2}\right)^{2}-\frac{1}{2} m^{2},
\end{aligned}
$$

где $q=\left\langle b_{i-}^{\dagger} b_{i+}\right\rangle=\left\langle b_{i+}^{\dagger} b_{i-}\right\rangle, \quad p=\left\langle b_{i+}^{\dagger} b_{j-}^{\dagger}\right\rangle=\left\langle b_{i+} b_{j-}\right\rangle$, $m=\left\langle b_{i+}^{\dagger} b_{i+}\right\rangle-\left\langle b_{i-}^{\dagger} b_{i-}\right\rangle$. Подчеркнем, что условие (7) также учитывается в приближении среднего поля.

Перейдя в $\mathbf{k}$-пространство и выполнив преобразование Боголюбова, получим диагонализованный гамильтониан

$$
H=\sum_{\mathbf{k} \alpha} \Omega_{\mathbf{k} \alpha} B_{\mathbf{k} \alpha}^{\dagger} B_{\mathbf{k} \alpha}+\frac{1}{2} \sum_{\mathbf{k} \alpha}\left(\Omega_{\mathbf{k} \alpha}-\Lambda_{\mathbf{k}}\right)+N C,
$$

где введены следующие обозначения:

$$
\begin{gathered}
\Omega_{\mathbf{k} \alpha}=\sqrt{\omega_{\mathbf{k}}^{2}+\lambda_{\mathbf{k}}^{2}+\tau^{2}+2 \varkappa_{\mathbf{k} \alpha}}, \quad \alpha= \pm \\
\omega_{\mathbf{k}}=\sqrt{\Lambda_{\mathbf{k}}^{2}-D_{\mathbf{k}}^{2}}, \quad \varkappa_{\mathbf{k} \pm}= \pm \sqrt{\omega_{\mathbf{k}}^{2} \lambda_{\mathbf{k}}^{2}+\Lambda_{\mathbf{k}}^{2} \tau^{2}} \\
\Lambda_{\mathbf{k}}=-v+\Delta+\frac{1}{2} Z V\left(1-b_{0}^{2}\right)-Z t^{m} b_{0}^{2} \gamma_{\mathbf{k}} \\
\lambda_{\mathbf{k}}=\mu-\frac{1}{2} Z V m+Z t^{l} b_{0}^{2} \gamma_{\mathbf{k}}, \\
D_{\mathbf{k}}=-\left(\frac{t^{p n} b_{0}^{2}}{2}+V p\right) Z \gamma_{\mathbf{k}}, \quad \tau=-Z t^{b} q \\
v\left(1-b_{0}^{2}\right)-\frac{1}{4} Z V\left(1-b_{0}^{2}\right)^{2}-\frac{1}{4} Z V m^{2}+Z V p^{2}+Z t^{b} q^{2} \\
\gamma_{\mathbf{k}}=\frac{1}{Z} \sum_{\langle\mathbf{r}\rangle} e^{i \mathbf{k r}}, \quad t^{m}=\frac{t^{p}+t^{n}}{2}, \quad t^{l}=\frac{t^{p}-t^{n}}{2}
\end{gathered}
$$

Дополнительные среднеполевые параметры $b_{0}$, $v, q, p, m$ определяются из условия минимума свободной энергии $F=N e_{0}-\frac{1}{\beta} \sum_{\mathbf{k}} \ln \left[1+n\left(\Omega_{\mathbf{k}-}\right)\right]$ $-\frac{1}{\beta} \sum_{\mathbf{k}} \ln \left[1+n\left(\Omega_{\mathbf{k}+}\right)\right], \quad$ где $\quad e_{0}=\frac{1}{2 N} \sum_{\mathbf{k} \alpha}\left(\Omega_{\mathbf{k} \alpha}-\Lambda_{\mathbf{k}}\right)+C$, $n\left(\Omega_{\mathbf{k} \alpha}\right)=1 /\left(\exp \beta \Omega_{\mathbf{k} \alpha}-1\right)$. После минимизации, мы получим следующую систему самосогласованных уравнений:

$$
2-b_{0}^{2}=\frac{1}{N} \sum_{\mathbf{k} \alpha} \Lambda_{\mathbf{k}}\left(1+\frac{\lambda_{\mathbf{k}}^{2}+\tau^{2}}{\varkappa_{\mathbf{k} \alpha}}\right) \frac{n\left(\Omega_{\mathbf{k} \alpha}\right)+\frac{1}{2}}{\Omega_{\mathbf{k} \alpha}},
$$

$$
\begin{aligned}
& v=\frac{Z}{N} \sum_{\mathbf{k} \alpha} \gamma_{\mathbf{k}}\left[\frac{t^{p n}}{2} D_{\mathbf{k}}-t^{m} \Lambda_{\mathbf{k}}+t^{l} \lambda_{\mathbf{k}}\right. \\
& \left.+\frac{\left(\frac{t^{p n}}{2} D_{\mathbf{k}}-t^{m} \Lambda_{\mathbf{k}}\right) \lambda_{\mathbf{k}}^{2}+t^{l} \omega_{\mathbf{k}}^{2} \lambda_{\mathbf{k}}-t^{m} \tau^{2} \Lambda_{\mathbf{k}}}{\varkappa_{\mathbf{k} \alpha}}\right] \frac{n\left(\Omega_{\mathbf{k} \alpha}\right)+\frac{1}{2}}{\Omega_{\mathbf{k} \alpha}}, \\
& q=\frac{1}{2 N} \sum_{\mathbf{k} \alpha} \tau\left(1+\frac{\Lambda_{\mathbf{k}}^{2}}{\varkappa_{\mathbf{k} \alpha}}\right) \frac{n\left(\Omega_{\mathbf{k} \alpha}\right)+\frac{1}{2}}{\Omega_{\mathbf{k} \alpha}}, \\
& p=-\frac{1}{2 N} \sum_{\mathbf{k} \alpha} D_{\mathbf{k}} \gamma_{\mathbf{k}}\left(1+\frac{\lambda_{\mathbf{k}}^{2}}{\varkappa_{\mathbf{k} \alpha}}\right) \frac{n\left(\Omega_{\mathbf{k} \alpha}\right)+\frac{1}{2}}{\Omega_{\mathbf{k} \alpha}}, \\
& m=-\frac{1}{N} \sum_{\mathbf{k} \alpha} \lambda_{\mathbf{k}}\left(1+\frac{\omega_{\mathbf{k}}^{2}}{\varkappa_{\mathbf{k} \alpha}}\right) \frac{n\left(\Omega_{\mathbf{k} \alpha}\right)+\frac{1}{2}}{\Omega_{\mathbf{k} \alpha}} .
\end{aligned}
$$

\section{4. Результаты}

В результате численного решения уравнений (13) были найдены дисперсионные зависимости энергии псевдомагнонов, а также зависимости критических температур $T_{c}$, при которых щель в спектре (псевдо)магнонов $\Omega_{\mathbf{k}}$ обращается в ноль, от псевдомагнитного поля $h$ (рис. $1-4)$. Зависимости $T_{c}(h)$ представляют собой фактически фазовые $T-h$ диаграммы.

На рис. 1 представлена фазовая $T_{c}-h$ диаграмма при различных значениях интеграла двухчастичного переноса (параметра биквадратичной двухцентровой анизотропии) $t^{b}$, при $\Delta=8, V=t^{p}=t^{n}=t^{p n}=1$. С увеличением псевдомагнитного поля $h$ при постоянной температуре $T$ щель в спектре возбуждений исчезает при $h=h_{c_{1}}$ и часть бозонов (псевдомагнонов) конденсируется, либо в точке $\mathbf{k}=(0,0)$, либо в $\mathbf{k}=(\pi, \pi)$, в зависимости от знака параметра одночастичного переноса $t^{p}$ (параметр ХY-анизотропии). Одновременно с этим, при $h>h_{c 1}$ возникает „Псевдонамагниченность“, как вдоль направления поля $m_{z}$, так и в ХY-плоскости $m_{x}$, которую можно определить через структурный фактор:

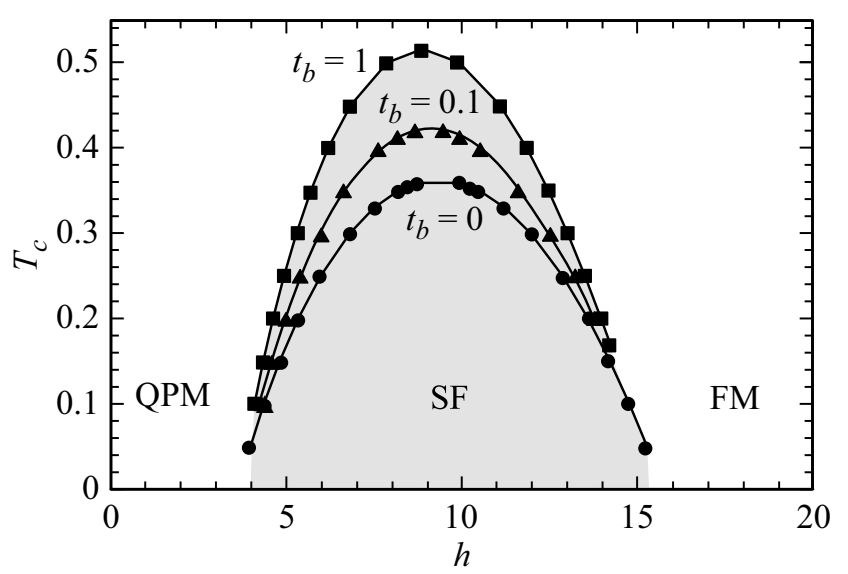

Рис. 1. Фазовая диаграмма $T_{c}-h$ при различных величинах интеграла двухчастичного переноса $t^{b} \quad(\Delta=8$, $\left.V=t^{p}=t^{n}=t^{p n}=1\right)$. 


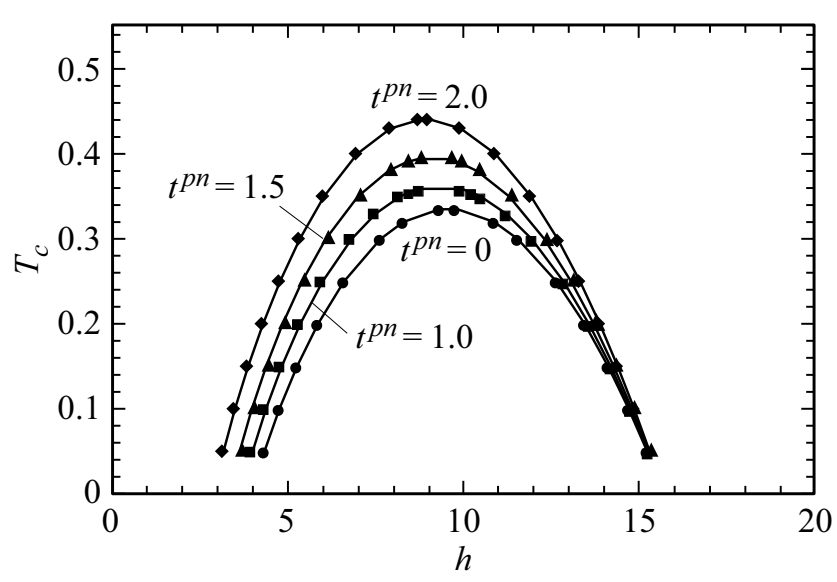

Рис. 2. Фазовая диаграмма $T_{c}-h$ при различных величинах интеграла одночастичного переноса $t^{p n}(\Delta=8$, $\left.V=t^{p}=t^{n}=1, t^{b}=0\right)$.

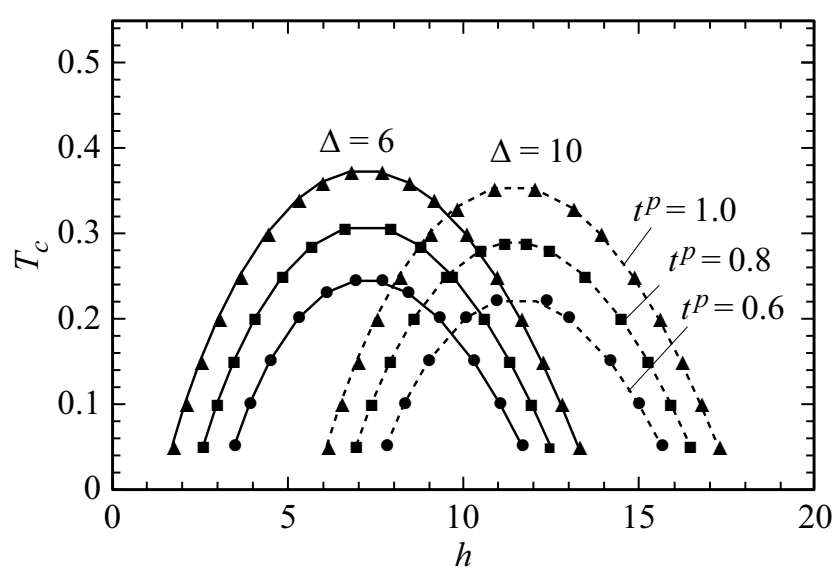

Рис. 3. Фазовая диаграмма $T_{c}-h$ при различных величинах интеграла одночастичного переноса $t^{p}$ и параметра одноцентровых корреляций $\Delta\left(V=t^{n}=t^{p n}=1, t^{b}=0\right)$.

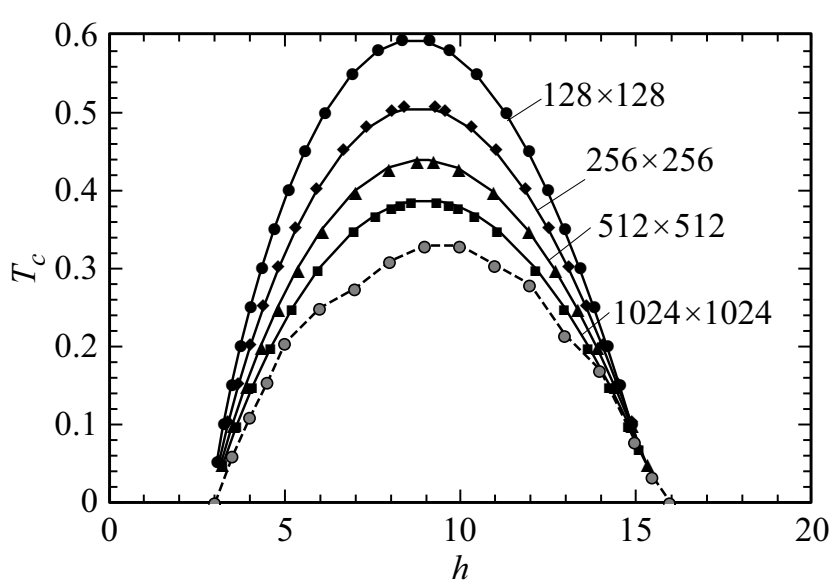

Рис. 4. Влияние размера решетки на фазовую диаграмму $T_{c}-h\left(\Delta=8, V=t^{p}=t^{n}=1, t^{p n}=2, t^{b}=0\right)$ и сравнение результатов с работой [17] (штриховая линия).
$N m_{x}^{2}=s(\pi)=\sum_{\mathbf{r}}(-1)^{\mathbf{r}}\left\langle S_{0 x} S_{\mathbf{r} x}\right\rangle$. При достижении следующего критического поля $h_{c 2}$, намагниченность в XYплоскости исчезает и отличной от нуля остается только компонента псевдоспинов вдоль $Z$-оси. Таким образом, рис. 1 фактически иллюстрирует зависимость температуры фазового перехода квантовый парамагнетик - сверхпроводящая XY-, или SF-фаза с отличными от нуля параметрами порядка типа $\left\langle S_{ \pm}\right\rangle$и/или $\left\langle S_{ \pm}^{2}\right\rangle$, сопровождаемая однородным ферро-упорядочением псевдоспинов вдоль $Z$-оси, от поля $h$. Температура фазового перехода растет с ростом интеграла двухчастичного переноса $t^{b}$, однако при низких температурах изменение $t^{b}$ практически не сказывается на величине критических полей.

На рис. 2 представлена фазовая $T_{c}-h$ диаграмма при различных значениях интеграла одночастичного переноса $t^{p n}$, инициирующего переходы $|0\rangle+|0\rangle \leftrightarrow|-1\rangle+|+1\rangle$, при $\Delta=8, \quad V=t^{p}=t^{n}=1$, $t^{b}=0$. Аналогично влиянию интеграла $t^{b}$, критическая температура $T_{c}$ растет с ростом $t^{p n}$, однако обратим внимание на заметный при этом эффект смещения поля $h_{c_{1}}$ в область малых полей и отсутствие заметного влияния на величину $h_{c_{2}}$, особенно в области низких температур.

Как показано на рис. 3, параметр одночастичного переноса $t^{p}, \quad$ отвечающий за переходы $|0\rangle+|+1\rangle \leftrightarrow|+1\rangle+|0\rangle$, играет ведущую роль в определении величин критических температур. На этом же рисунке представлены фазовые $T_{c}-h$ диаграммы при двух различных значениях параметра одноцентровых корреляций. Очевидно, что рост параметра $\Delta$ приводит к смещению фазовых диаграмм в область больших псевдомагнитных полей.

Фазовые диаграммы, представленные на рис. 1-3, получены в результате численных расчетов на квадратной решетке с размером $512 \times 512$ со свободными границами. Влияние размера решеток показано на рис. 4 для значения параметров упрощенного гамильтониана, принятых в работе [17]. Для сравнения штриховой линией приведены результаты континуального предела работы [17]. Существенная роль размера решетки связана с особенностями определения точки фазового перехода как точки обращения в нуль щели в спектре псевдомагнонов, точность определения которой зависит от выбранного размера решетки.

\section{5. Заключение}

В рамках (псевдо)спинового формализма была изучена двумерная анизотропная система $S=1$-центров типа зарядовых триплетов в системах с переменной валентностью или систем „Полужестких“ бозонов с ограничением на заполнение узлов решетки $n=0,1,2$. Используя представление швингеровских бозонов, нами были найдены условия, при которых система переходит в сверхпроводящее состояние. Установлено, что определяющую роль в формировании данной фазы играет одночастичный перенос РP-типа, тогда как транспорт PN-типа 
способствует образованию фазы в области меньших полей, а двухчастичный перенос повышает температуру фазового перехода.

\section{Список литературы}

[1] R.J. Birgeneau, J. Skalyo Jr., G. Shirane. J. Appl. Phys. 41, 1303 (1970).

[2] M. Steiner, K. Kakurai, J.K. Kjems, D. Petitgrand, R. Pynn. J. Appl. Phys. 61, 3953 (1987).

[3] B. Dorner, D. Visser, U. Steigenberger, K. Kakurai, M. Steiner. Z. Phys. B: Condens. Matter 72, 487 (1988).

[4] J.L. Manson, A.G. Baldwin, B.L. Scott, J. Bendix, R.E. Del Sesto, P.A. Goddard, Y. Kohama, H.E. Tran, S. Ghannadzadeh, J. Singleton, T. Lancaster, J.S. Moller, S.J. Blundell, F.L. Pratt, V.S. Zapf, J. Kang, C. Lee, M.-H. Whangbo, C. Baines. Inorg. Chem. 51, 7520 (2012).

[5] K. Wierschem, P. Sengupta. Mod. Phys. Lett. B 28, 1430017 (2014).

[6] A. Paduan-Filho, X. Gratens, N.F. Oliveira. Phys. Rev. B 69, 020405 (2004).

[7] A.S. Moskvin. JETP 121, 477 (2015).

[8] A.S. Moskvin. Phys. Rev. B 84, 075116 (2011).

[9] A.S. Moskvin. J. Phys.: Condens. Matter 25, 085601 (2013).

[10] A.S. Moskvin. J. Phys.: Conf. Ser. 592, 012076 (2015).

[11] E. Dagotto, A. Moreo. Phys. Rev. Lett. 63, 2148 (1989).

[12] J. Richter, J. Schulenburg. Eur. Phys. J. B 73, 117 (2010).

[13] R.R.P. Singh, W. Zheng, J. Oitmaa, O.P. Sushkov, C.J. Hamer. Phys. Rev. Lett. 91, 017201 (2003).

[14] J. Reuther, P. Wölfle. Phys. Rev. B 81, 144410 (2010).

[15] L. Siurakshina, D. Ihle, R. Hayn. Phys. Rev. B 64, 104406 (2001).

[16] S. Sachdev, R.N. Bhatt. Phys. Rev. B 41, 9323 (1990).

[17] H.T. Wang, Y.Wang. Phys. Rev. B 71, 104429 (2005).

[18] H. Chen, L. Yu, Z. Su. Phys. Rev. B 48, 12692 (1993).

[19] H. Wangand, J.S.K. Li, Z. Su. Phys. Rev. B 59, 12805 (1994).

[20] H. Xingand, G. Su, S. Gao, J. Chu. Phys. Rev. B 66, 054419 (2002).

[21] A.S.T. Pires, L.S. Lima, M.E. Gouvea. J. Phys. Condens. Matter 20, 015208 (2008).

Редактор Т.Н. Василевская 